\title{
Dynamic fracture instability of tough bulk metallic glass
}

\author{
J. X. Meng, ${ }^{1}$ Z. Ling, ${ }^{1}$ M. Q. Jiang, ${ }^{1}$ H. S. Zhang, ${ }^{1}$ and L. H. Dai ${ }^{1,2, a)}$ \\ ${ }^{1}$ State Key Laboratory of Nonlinear Mechanics, Institute of Mechanics, Chinese Academy of Science, \\ Beijing 100190, People's Republic of China \\ ${ }^{2}$ State Key Laboratory of Explosion Science and Technology, Beijing Institute of Technology, Beijing \\ 100081, People's Republic of China
}

(Received 18 March 2008; accepted 27 March 2008; published online 2 May 2008)

\begin{abstract}
We report the observations of a clear fractographic evolution from vein pattern, dimple structure, and then to periodic corrugation structure, followed by microbranching pattern, along the crack propagation direction in the dynamic fracture of a tough $\mathrm{Zr}_{41.2} \mathrm{Ti}_{13.8} \mathrm{Cu}_{12.5} \mathrm{Ni}_{10} \mathrm{Be}_{22.5}$ (Vit.1) bulk metallic glass (BMGs) under high-velocity plate impact. A model based on fracture surface energy dissipation and void growth is proposed to characterize this fracture pattern transition. We find that once the dynamic crack propagation velocity reaches a critical fraction of Rayleigh wave speed, the crack instability occurs; hence, crack microbranching goes ahead. Furthermore, the correlation between the critical velocity of amorphous materials and their intrinsic strength such as Young's modulus is uncovered. The results may shed new insight into dynamic fracture instability for BMGs. (C) 2008 American Institute of Physics. [DOI: 10.1063/1.2913206]
\end{abstract}

The instability of dynamic cracks is of both fundamental and practical importance. ${ }^{1-5}$ In tensile fracture, a propagating crack will oscillate and branch as its speed reaches a critical fraction of Rayleigh wave velocity $V_{R}$. This crack instability brings out special pattern sequence known as "mirror, mist, and hackle." An initially featureless mirrorlike fracture surface begins to appear misty and then evolves into a rough hackle region. ${ }^{6-8}$ Out of this recognition, it was that the quest for a more clearly physical failure process led to the study of brittle glassy polymers (PMMA), oxide glasses, Homalite100 , and polymer gels ${ }^{2,6,7,9-12}$ for years. When the crack tip of brittle materials becomes unstable, microbranchings are generally observed at velocities about $0.3-0.6 V_{R}$. However, the dynamic instability mechanics are still experimentally and theoretically an open question. Recently, brittle and lessbrittle bulk metallic glasses ${ }^{13-18}$ (BMGs) have been used as another kind of model materials generating much interest. Self-corrugation characteristic of nanoscale dimples and strips ${ }^{13-18}$ is quite well exhibited by extension from preexisting notch of Mg-based BMGs, Fe-based BMGs, and Ni-based BMGs. Particularly, Wang et al. found an unusual fractographic evolution from dimple structure to periodic corrugation pattern and then to mirror zone along the crack propagation direction in dynamic fracture of a brittle Mg-based BMG. ${ }^{18}$ It is noted that no microbranching morphology has been observed in these brittle and less-brittle BMGs. One question naturally arises. Does the crack microbranching-based dynamic fracture instability exist in BMGs? In this letter, we report a clear fractographic evidence to demonstrate the microbranching pattern in the fracture surface of a tough $\mathrm{Zr}$-based BMG subjected to high speed plate impact. Possible origin of the observed dynamic fracture instability in BMGs was discussed as well.

In order to study tough BMGs' dynamic fracture instability, we choose $\mathrm{Zr}_{41.2} \mathrm{Ti}_{13.8} \mathrm{Cu}_{12.5} \mathrm{Ni}_{10} \mathrm{Be}_{22.5}$ (Vit.1) with the fracture toughness $K_{C}>60 \mathrm{MPa} \sqrt{\mathrm{m}}$ as a model material, which exhibits better tough behavior ${ }^{19,20}$ than Mg-based brittle BMGs with much lower $K_{C}<2 \mathrm{MPa} \sqrt{\mathrm{m}} .{ }^{13,16}$ The

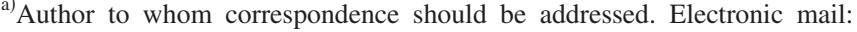
lhdai@1nm.imech.ac.cn.
}

samples before high-speed impact experiments were identified to be full amorphous state by conventional x-ray diffraction. Plate impact experiments were performed on the onestage light gas gun. The Vit.1 BMG samples used in the present study were machined into the disks that here $8 \mathrm{~mm}$ in diameter and $0.3 \mathrm{~mm}$ in thickness, which were sandwiched between the front and the base steel plates being of higher wave impedance than Vit.1. The launched aluminum flyer plate at the speed of $500 \mathrm{~m} / \mathrm{s}$ was impacted onto the front steel plate. At the moment of impact, the generated planar compressive shock wave in the front steel plate passes through the Vit.1 samples and induces a circumferential tensile stress of about $2.3 \mathrm{GPa}$ on the free edge of the sample. This circumferential tensile stress exceeds the tensile strength of Vit.1 with $\sigma_{T} \sim 1.9 \mathrm{GPa}$ (Ref. 21) and thus leads to a mode-I crack at the edge propagating along the radial direction which finally separates the sample into several small pieces [as shown in Fig. 1(a)].

When examining the fracture surfaces of the loaded samples with a high resolution scanning electronic microscopy (HRSEM) (FEI-Sirion NC HRSEM with $1.5 \mathrm{~nm}$ resolution), clear nanoscale structures and their transition processes are observed on the fracture surface, as shown in Fig. 1. Figure 1(b), corresponding to the rectangle mark in Fig. 1(a), displays a fractographic transition from vein pattern to dimple structure then to periodic corrugation pattern, followed by microbranching zone along the crack propagation direction in the dynamic fracture. Four zones as shown in Fig. 1(b) from A to D, as the arrow illustrated, are divided and their enlarged images are clearly shown in Figs. 1(c)-1(f). Their characteristic length scales along the crack propagation direction are measured and plotted in Fig. 2. The first zone is typical vein pattern, as shown in Fig. 1(c), with equivalent diameter about $500 \mathrm{~nm}$. It is noted that these veins are in approximately equiaxial shape, which means that the tensile stress plays a dominant role in the edge zone and mode-I cracking does initiate. Following the first vein pattern zone are the dimple structures, as shown in Fig. 1(d), with characteristic size linearly decreasing from approximately 140 to $80 \mathrm{~nm}$ [see Fig. 2 region II]. With the crack propagating, the surface shows a typical flat mirror zone, but when 


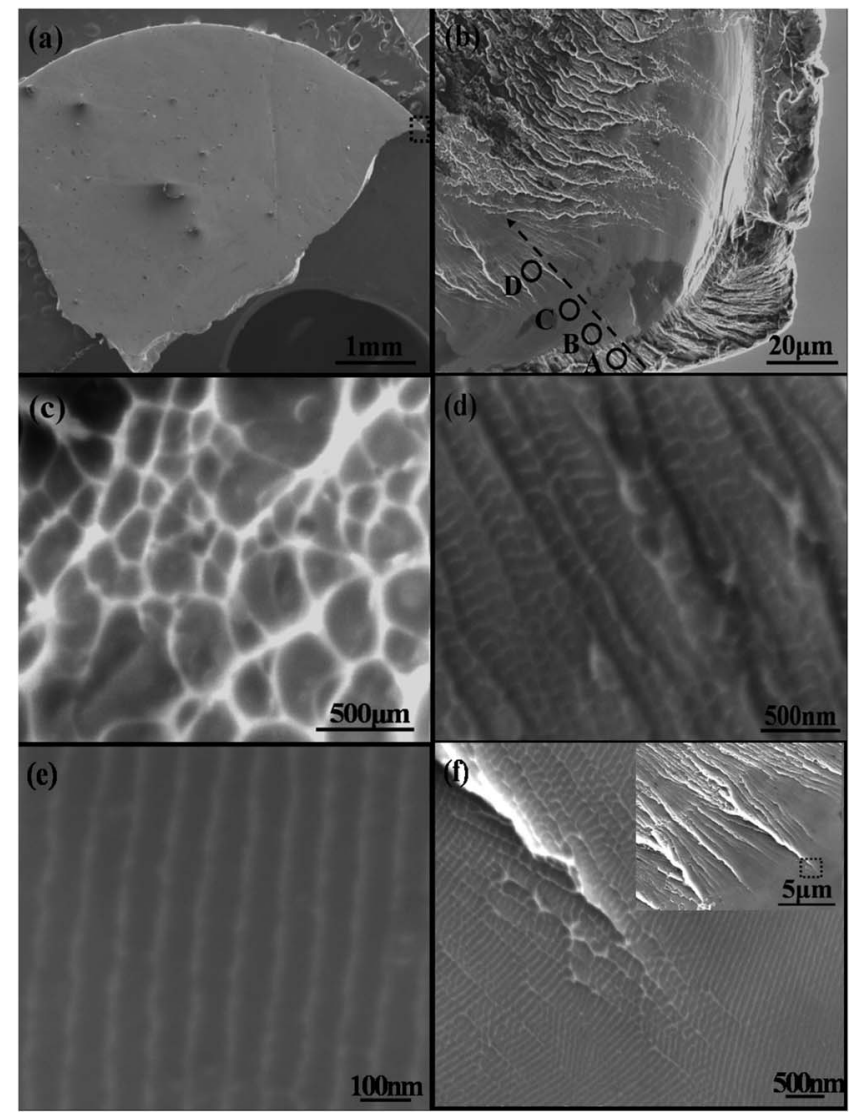

FIG. 1. The fracture features of the (Vit.1) BMGs' fracture surface by SEM. (a) The marcomorphology of one separated piece. (b) The macromorphologies evolution in the fracture surface. (c) Vein patterns. (d) Dimples structures. (e) Periodic corrugations. (f) Microbranching of the marked region of the inset (inset showing the whole feature).

we enlarged the zone by HRSEM the periodic corrugation patterns which are perpendicular to the crack propagate direction, as shown in Fig. 1(e), replace the dimple structure and become dominant. It is worth pointing out that the regular nanoscale wavy corrugations' average spacing is about $70 \mathrm{~nm}$ and total propagate distance is $\sim 17 \mu \mathrm{m}$. This result indicates that the crack propagates stably in this zone where the fracture surface energy flux and dissipation are on a bal-

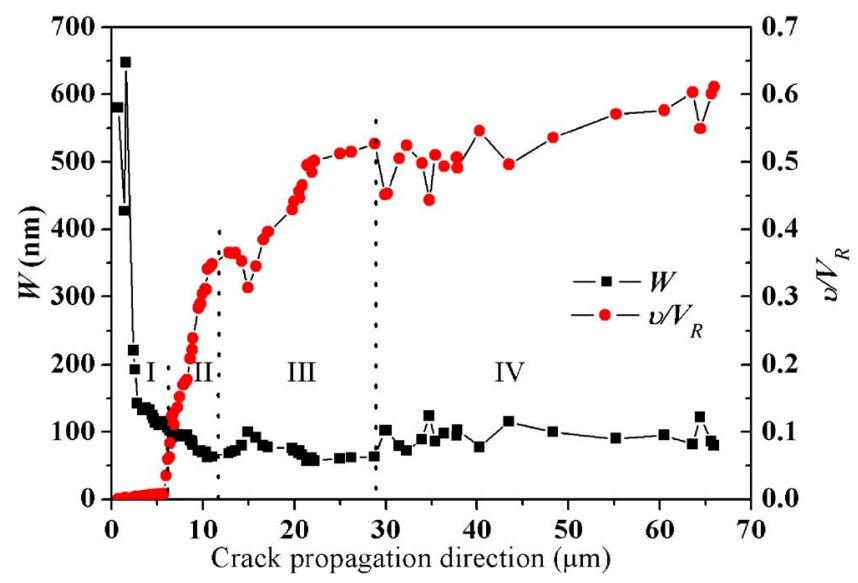

FIG. 2. (Color online) Characteristic width $(w)$ of the micro structures and the ratio of crack propagating velocity to Rayleigh wave speed $\left(v / V_{R}\right)$ along the crack propagate process in the fracture surface. Regions I, II, III and IV correspond to the vein patterns, dimple structures, periodic corrugations, and

microbranching zone, respectively.
Downloaded 30 Jul 2009 to 159.226 .231 .70 . Redistribution subject to AlP license or copyright; see http://apl.aip.org/apl/copyright.jsp ance. However, with the crack propagation speed increasing, the fracture surface becomes rough and the crack traveling mode changes, consequently, a new zone appears. In this zone, microbranching together with periodic corrugation become dominant morphology, as shown in Fig. 1(f), and the periodic corrugation's characteristic width undulates between 80 and $120 \mathrm{~nm}$ [see Fig. 2 region IV]. It is important that microbranching becomes dominant [see the inset of Fig. 1(f)] because it promotes the increased kinetic energy dissipation of the fast running dynamic crack and produces more fracture surfaces. The observations of clear fractographic evolution provide powerful evidence for the dynamic fracture instability along the crack propagation direction of Zr-based BMGs.

Solving dynamic crack problems is in general extremely difficult due to the solution correlating with material intrinsic properties. ${ }^{22}$ Up to now, only some special problems have been solved. To understand the correlation, we consider that this dynamic crack is a single edge crack accompanied with continuous void growth process in a semi-infinite body. According to the linear elastic fracture mechanics, ${ }^{1,3}$ the crack propagation velocity can be written as $v=V_{R}[1$ $\left.-\Gamma(v) E / K_{I}(v)^{2}\right]$, where $K_{I}(v)$ is the dynamic stress intensity and $\Gamma(v)$ is the fracture surface energy of the material. The dynamic stress intensity factor $K_{I}(v)$ can be characterized by $K_{I}(v)=k(v) K_{I}^{s}$, where the crack velocity coefficient $k(v)$ $=\left(1-v / V_{R}\right)\left(\right.$ Refs. 1 and 23) and $K_{I}^{s}$ is the static stress intensity factor which is given by $K_{I}^{S}=1.12 \sigma \sqrt{\pi l}$, , ${ }^{1,8}$ where $l$ is the crack propagation distance from the sample edge. In view of this problem, it should be pointed out that it is a continuous changing process of the characteristic length scales of the nanoscale structures that relate to the fracture surface energy flux and dissipation. In tension, void growth and linkage would be facilitated by a tensile stress state, perhaps leading to premature fracture. According to Wright et al., ${ }^{24}$ voids are predicted to spontaneously form from the coalescence of free volume. These voids are then expected to coarsen and the fracture surface energy fluctuates with these voids. According to their work, ${ }^{24}$ the fracture surface energy can be estimated by $\Gamma(v)=r(v) \mu / 2 \Omega$, where $r(v)$ is taken as the half of the characteristic width of the fracture surface patterns, $\mu$ is the free volume energy chemical potential about $10^{-18} \mathrm{~J}$, and $\Omega$ is the average atomic volume. ${ }^{25}$ Then we have the equation, $v / V_{R}=1-\left[(r(v) \mu E) /\left(2.51 \Omega \sigma^{2} \pi l\right)\right]^{1 / 3}$. After calculating, we get the evolution of $v / V_{R}$ along the crack propagation direction, which is shown in Fig. 2. We find that on the stages of dimple and periodic corrugation, $v / V_{R}$ sharply increase from 0.01 to 0.52 , and when $v / V_{R} \geqslant 0.52$ the nanoscale periodic corrugation pattern becomes unstable and microbranching, presenting the fracture dynamic instability, occurs.

The fractaographic evolution in most brittle glassy materials exhibit a characteristic value of $v / V_{R}$ when a highly rough hackled region forming for violent oscillation to dissipation more fracture surface energy. Schardin and Struth ${ }^{11}$ found that when the critical velocity $v$ of oxide glass reaches around $0.51 V_{R}$ (Refs. 9-11), the crack microbranching sets in. Ravi-Chander and Knauss made a systematic study on the dynamic fracture about Homalite- $100^{6}$ and found critical value $v / V_{R}$ is about 0.40 . Fineberg ${ }^{12}$ and Sharon et al. ${ }^{7}$ found the critical velocities $v / V_{R}$ are about 0.36 and 0.44 for PMMA and soda lime, respectively, and the universality of 


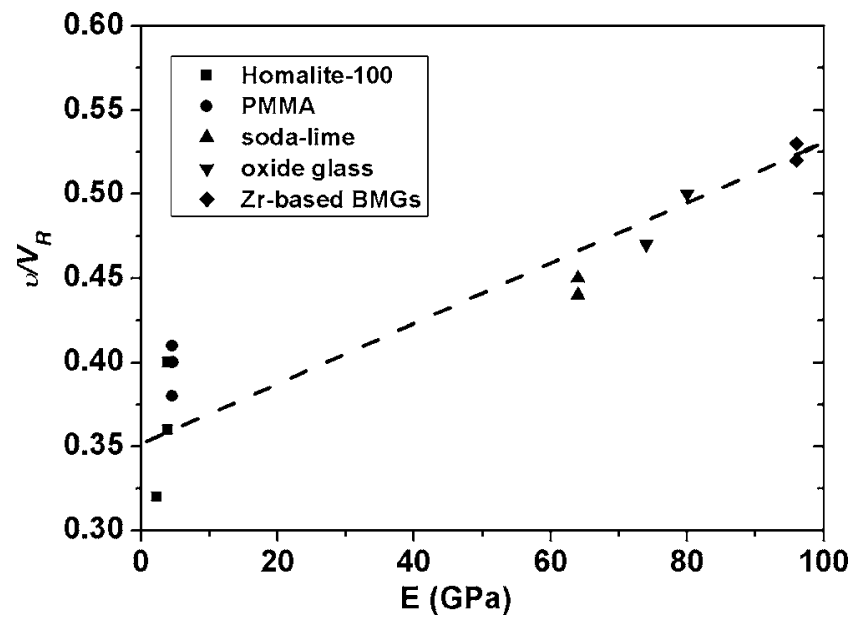

FIG. 3. The critical velocity of amorphous materials, such as PMMA, Homaite-100, soda lime, oxide glass, and Zr-based BMGs are between $0.30-0.60 \mathrm{~V}_{\mathrm{R}}$, and $v / V_{R}$ increase with their Young's modulus.

microbranching instability was discussed. The above branching instability and the undulation of fracture surface energy have a similarity among PMMA, Homalite-100, glass, and BMGs, which leads to the conclusion that the microbranching instability is a universal phenomenon in the dynamic fracture of amorphous materials. Although the microstructure of the materials may change some features of the instability's evolution, the basic phenomenon is the same. As far as the above amorphous materials, a propagating crack can easily deviate from its original plane and thus forms a rough fracture surface due to the absence of long-range order ${ }^{26}$ and the critical speed is not a universal constant but material dependent. ${ }^{22}$ In this connection, a linear correlation of the ratio of $v / V_{R}$ and materials' intrinsic strength such as Young's modulus is observed, as shown in Fig. 3. This result indicates that a dynamic crack can be viewed as a definite state of energy system and the fractographic transition with the energy changing by its flux and dissipation, when the energy surpasses a critical which connect with material's intrinsic strength, a correlated instability of microbranching occurs in materials' dynamic fracture surface.

In summary, the fracture surface of a tough Vit.1 under high-speed impact presents an interesting nanoscale structural evolution from vein pattern to dimple and then to periodic corrugation, followed by crack microbranching pattern. This suggests that dynamic crack in tough BMGs has a clear transition process owing to the crack dynamic instability, particularly forming the microbranching morphology to dissipating extra energy in the fracture surface. To rationalize this instability phenomenon, a model based on fracture surface energy dissipation and void growth in fracture surface is developed. The results indicate that once the crack velocity reaches a critical, the crack instability occurs and then microbranching goes ahead. When extending to other amorphous materials, a correlation between the ratio of $v / V_{R}$ and intrinsic strength, such as Young's modulus, is revealed. We believe that these findings are of importance for understanding relationship between energy dissipation and microstructure as well as crack microbranching-based dynamic fracture instability in BMGs.

The authors are grateful to Professor B. C. Wei for his assistance in material preparation and acknowledge the financial support from the Natural Science Foundation of China (Grants Nos. 10725211and 10721202) and the key project of Chinese Academy of Sciences (Nos. KJCX-SWL08 and KJCX2-YW-M04).

${ }^{1}$ L. B. Freund, Dynamic Fracture Mechanics (Cambridge University Press, Cambridge, 1990).

${ }^{2}$ M. A. Meyers, Dynamic Behavior of Materials (University of California, San Diego, 1994).

${ }^{3}$ M. Martin, N. N. Thadhani, L. Kecskes, and R. Dowding, Scr. Mater. 55, 1019 (2006).

${ }^{4}$ R. D. Conner, A. J. Rosakis, W. L. Johnson, and D. M. Owen, Scr. Mater. 37, 1373 (1997)

${ }^{5}$ A. Livne, O. Ben-David, and J. Fineberg, Phys. Rev. Lett. 98, 124301 (2007).

${ }^{6}$ K. Ravi-Chandar and W. G. Knauss, Int. J. Fract. 25, 247 (1984).

${ }^{7}$ E. Sharon, G. Cohen, and J. Fineberg, Phys. Rev. Lett. 88, 085503 (2002).

${ }^{8}$ B. Lawn and T. R. Wilshaw, Fracture of Brittle Solids, 2nd ed. (Cambridge University Press, Cambridge, 1993).

${ }^{9}$ F. P. Bowden, J. H. Brunton, J. E. Field, and A. D. Heyes, Nature (London) 216, 38 (1967).

${ }^{10}$ H. E. Edgerton and P. E. Barstow, J. Am. Ceram. Soc. 24, 131 (1941).

${ }^{11}$ H. Schardin and W. Struth, Glastech. Ber. 16, 219 (1938).

${ }^{12}$ J. Fineberg, S. P. Gross, M. Marder, and H. L. Swinney, Phys. Rev. Lett. 67, 457 (1991).

${ }^{13}$ X. K. Xi, D. Q. Zhao, M. X. Pan, W. H. Wang, Y. Wu, and J. J. Lewandowski, Phys. Rev. Lett. 94, 125510 (2005).

${ }^{14}$ G. Wang, Y. T. Wang, Y. H. Liu, M. X. Pan, D. Q. Pan, and W. H. Wang, Appl. Phys. Lett. 89, 121909 (2006).

${ }^{15}$ Z. F. Zhang, F. F. Wu, W. Gao, J. Tan, and Z. G. Wang, Appl. Phys. Lett. 89, 251917 (2006).

${ }^{16}$ G. Wang, D. Q. Zhao, H. Y. Bai, M. X. Pan, A. L. Xia, B. S. Han, X. K. Xi, Y. Wu, and W. H. Wang, Phys. Rev. Lett. 98, 235501 (2007).

${ }^{17}$ J. Shen, W. Z. Liang, and J. F. Sun, Appl. Phys. Lett. 89, 121908 (2006).

${ }^{18}$ G. Wang, X. H. Xu, Y. N. Han, B. S. Han, F. J. Ke, and W. H. Wang, "Ductile to brittle transition in dynamic fracture of a brittle bulk metallic glass," Phys. Rev. Lett. (submitted).

${ }^{19}$ P. Lowhaphandu and J. J. Lewuandowski, Scr. Mater. 38, 1811 (1998).

${ }^{20}$ L. F. Liu, L. H. Dai, Y. L. Bai, and B. C. Wei, J. Non-Cryst. Solids 351, 3259 (2005).

${ }^{21}$ J. Lu, G. Ravichandran, and W. L. Johnson, Acta Mater. 51, 3429 (2003).

${ }^{22}$ K. Ravi-Chandar, Int. J. Heat Mass Transfer 90, 83 (1998).

${ }^{23}$ H. Gao, J. Mech. Phys. Solids 41, 457 (1993).

${ }^{24}$ W. J. Wright, T. C. Hufnagel, and W. D. Nix, J. Appl. Phys. 93, 1432 (2003).

${ }^{25}$ Y. Zhang and A. L. Greer, J. Alloys Compd. 434-435, 2 (2007).

${ }^{26}$ T. Cramer, A. Wanner, and P. Gumbsch, Phys. Rev. Lett. 85, 788 (2004). 